\title{
A NEW HANDHELD SCANNER FOR 3D SURVEY OF SMALL ARTIFACTS: THE STONEX F6
}

\author{
G. Patrucco ${ }^{1, *}$, F. Rinaudo ${ }^{1}$, A. Spreafico ${ }^{1}$ \\ ${ }^{1}$ DAD, Department of Architecture and Design, Politecnico di Torino, Viale Mattioli 39, 10125 Torino \\ (giacomo.patrucco, fulvio.rinaudo, alessandra.spreafico)@polito.it
}

Commission II, WG II/8

KEY WORDS: 3D Model, close-range photogrammetry, structured light laser scanner, movable heritage, Cultural Heritage documentation

\begin{abstract}
:
Movable heritage preserved in our museums are an invaluable evidence of our past. In order to properly respond to the need of 3D documentation of these significant assets, in the last few years both range-based and image-based solutions have been developed by researchers operating in the framework of Geomatics with a special focus on reaching a high level of detail and on texture radiometric quality, taking into consideration the intrinsic fragility of these kinds of objects which during the survey require a contactless approach. During the presented research a collection of architectural models representing ancient Nubian temples from "Museo Egizio di Torino" had been digitalized using different techniques; in particular, the wooden maquette of the temple of El-Hilla has been acquired using a new structured light handheld laser scanner, the Stonex F6 SR, and applying a close-range photogrammetric approach. In this paper a comparison between the two approaches is proposed as regards acquisition workflow, final results and suitability as regards digitisation of objects belonging to movable heritage and museum collections.
\end{abstract}

\section{INTRODUCTION}

3D modelling of Cultural Heritage related objects is becoming more and more relevant in the framework of Geomatics researches and entails issues connected to the faithfulness of these 3D models describing the real object shapes by considering the final user needs, and the fruition requirements. The possibilities related to management of 3D databases and online visualisation tools to allow the sharing of information make these kinds of 3D models, obtained with Geomatics techniques, a valuable tool for the documentation of Cultural Heritage. The documentation of Cultural Heritage assets is becoming even more necessary and useful for valorisation purposes of not only built heritage, but also the so-called movable heritage, specifically museum exhibits. These kinds of assets are constantly exposed to several risks. In many cases, some artworks are not accessible to the public, some of these collections often remain stored in warehouses, they are not studied and their state of conservation is unknown.

In the last few years, Geomatics has developed new imagebased and range-based techniques and strategies for digitalising museum artworks (Adami et al. 2015; Donadio et al. 2018, Balletti et al. 2018), in order to answer to the need of documentation. More and more frequently research activities are being carried out to produce high-resolution 3D digital replicas of objects belonging to museum collections (Guidi et al. 2017; Patrucco et al. 2018), contributing not only to metric documentation but also to cultural dissemination of these inaccessible masterpieces. One of the most commonly used technique is the close-range photogrammetry (Povroznik 2018), that in the last years exploits macro lens for digitization of small objects especially in the field of archaeology (Marziali $\&$ Dionisio 2017). The development of new photogrammetric computer vision technologies and the improvements in imagematching algorithms allow to achieve 3D models with a very high level of detail, furthermore the opportunity to generate a high-resolution photographic texture allows to document also the consistency of the modelled object making this technique very suitable for digitisation of museum collections (Donadio 2018). Recently, technologies based on triangulation and structured light laser scanners are becoming more frequently used in the field (Dondi et al. 2017; Kersten et al. 2018). These kinds of instruments, especially handheld solutions, allow to acquire a large amount of data very quickly and with submillimetre accuracy; furthermore, many devices have implemented the possibility of generating a photographic texture in order to provide not only the geometry of the measured objects, but also their radiometry (Allegra et al. 2017).

In detail, the paper introduce the new F6 family of handheld laser scanners based on structured light technology recently developed by Mantis Vision and proposed as fast solutions to scan objects. In particular, the F6 Short Range (SR) is especially recommended for small artefacts as little statues or furniture. The present research is a former attempt to test and evaluate the competitiveness of the F6 SR as handheld 3D laser-based solution to digitize museum collection comparing this solution with a more traditional (and well known in terms of achievable precision) approach like the photogrammetric one.

\section{MODEL OF THE TEMPLE OF EL-HILLA}

The research takes place in the framework of B.A.C.K. TO T.H.E. F.U.T.U.RE. Project (BIM Acquisition as Cultural Key TO Transfer Heritage of ancient Egypt For many Uses To many Users REplayed) carried out by Politecnico di Torino (Lo Turco et al. 2018). One of the main goals of the project aims to create $3 \mathrm{D}$ databases of a collection of wooden maquettes dated back to the XVIII century and belonging to the "Museo Egizio" of Torino. These artefacts constitute valuable witnesses of the ancient Nubian temples moved from

\footnotetext{
* Corresponding author
} 
their original placements because of the construction of the Aswan dam. A very sensitive aspect of this research project is represented by the realisation of reality-based 3D models of the maquettes with high level of detail, to provide both geometric and radiometric information about the real object. This kind of objects constitute interesting case studies for the present investigation because of their complexity. In fact, they are miniature architectures with tiny decorations, realised by using fragile materials and articulated surfaces. Furthermore, the majority of the maquettes are composed by two halves allowing to see the inner configuration of the temple, also composed by inner chambers uneasy to survey. The models cannot be touched due to the fragility of the materials. For this reason, they have been recorded at Museo Egizio by the Geomatics Lab for Cultural Heritage of the Politecnico di Torino, applying both image and range-based approach to obtain two different texturized point clouds of the wooden maquettes. The present analysis considers the right part of the Temple of El-Hilla ( Figure 1) as case study whose dimensions are approximatively $80 \times 22 \times 30 \mathrm{~cm} 3$.

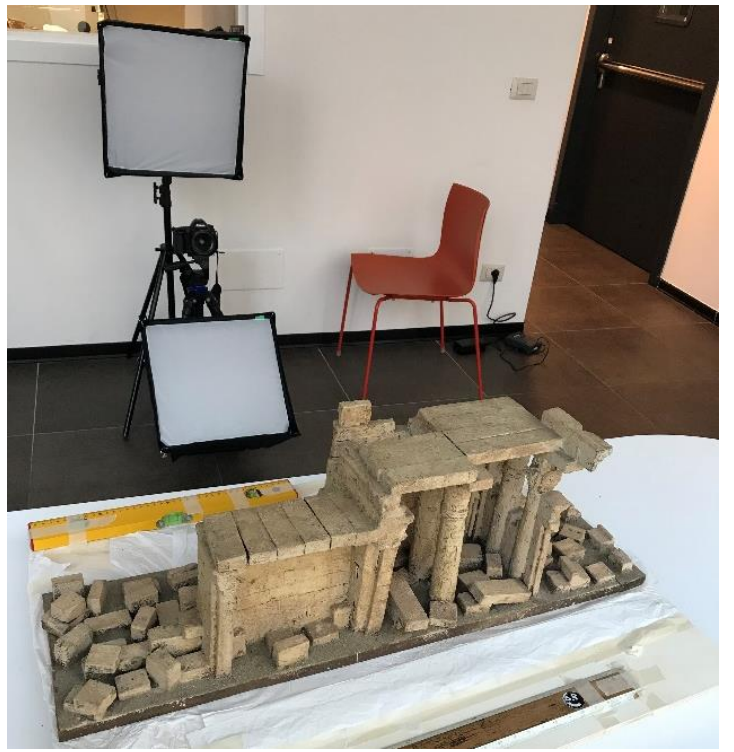

Figure 1. The Temple of El-Hilla during the photogrammetric set of acquisition

\section{DATA ACQUISITION}

A complete photogrammetric acquisition has been performed with a high-resolution full frame digital camera, and the aforementioned F6 SR structured light scanner.

\subsection{Digital images acquisition}

The Canon EOS 5DSR, a full-frame high-resolution DSLR camera (Table 1), has been used with Zeiss Milvus $50 \mathrm{~mm} \mathrm{f} / 2 \mathrm{M}$ macro lens mounted on it (Figure 2) to perform the close-range photogrammetric image acquisition. A photographic tripod and a rotating platform have been used to ease the stereoscopic image acquisition. The architectural model has been placed on the platform and rotated at regular intervals in order to acquire overlapped images of the object. Three scale bars have been placed on the platform and fixed with adhesive tape at the sides of the wooden maquette, in order to provide a metric reference allowing to scale the model during the subsequent phase of data processing. To avoid, as much as possible, shadows or blur effects, two LED panels (by LUPO) has been positioned on the sides of the camera during the acquisition phase, to provide a homogeneous illumination. Four rings of images have been acquired: an inclined acquisition from the top (to cover the upper parts of the model), two with semi-forward configuration (in order to reach a good coverage of the walls and vertical elements of the maquette) and another one inclined from the bottom to acquire the ceilings of the colonnades.

\begin{tabular}{|l|l|}
\hline Model & Canon EOS 5DSR \\
\hline Sensor & CMOS 50.3 [Mpx] \\
\hline Sensor size & $36 \times 24[\mathrm{~mm}]$ \\
\hline Image size & $8688 \times 5792[\mathrm{px}]$ \\
\hline Lens & Zeiss Milvus 50mm f/2M \\
\hline Focal length & $50[\mathrm{~mm}]$ \\
\hline
\end{tabular}

Table 1. Canon EOS 5DSR main specifications
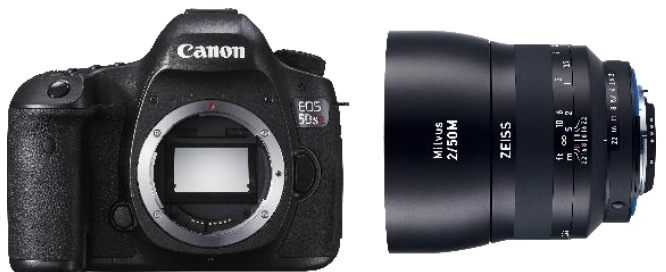

Figure 2. On the left, the full-frame high resolution DSLR camera used during data acquisition (Canon EOS 5DSR). On the right, Zeiss Milvus $50 \mathrm{~mm} \mathrm{f} / 2 \mathrm{M}$ macro lens

In Figure 3 it is possible to observe the acquisition asset schemes.

A dataset of 126 digital images has been acquired from an average shooting distance of $1.5 \mathrm{~m}$. The whole image acquisition process took of about 35 minutes by considering also the time required for the changes of the camera assets and lighting conditions configuration, in addition to the effective time needed to record the digital images.

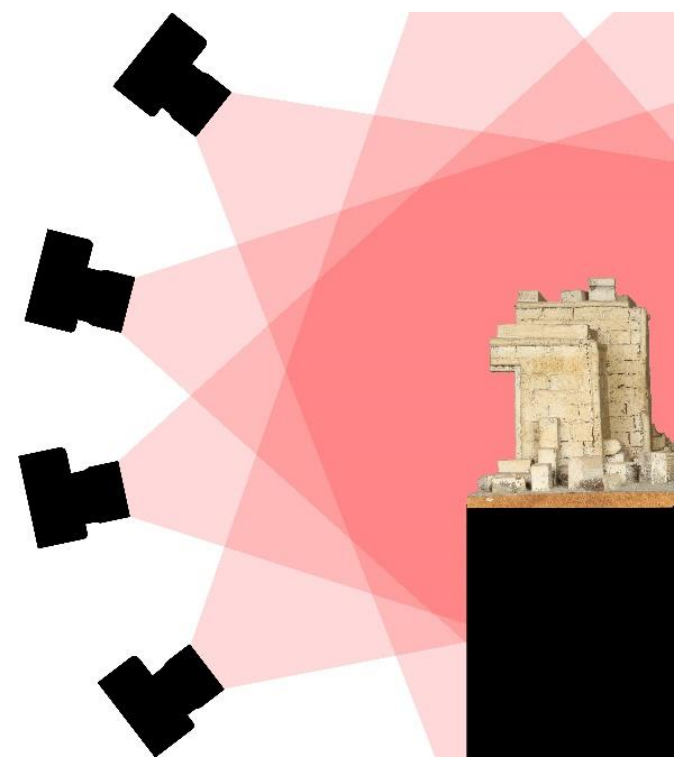

Figure 3. Scheme of the acquisitions (the four assets of the camera can be observed)

\subsection{Range-based acquisition: the Stonex F6 SR}

The handheld F6 SR structured-light laser scanner (Figure 4), along with the F6 SMART, constitutes the new version (sold from 2018 by Stonex) of the previous F5 laser scanner family (Kersten et al. 2018); the F6 SMART working at a larger distance is used to survey bigger elements, as cars or also entire 
rooms, while the technical specifications (Table 2) of the F6 SR depict an interesting tool to survey small objects.

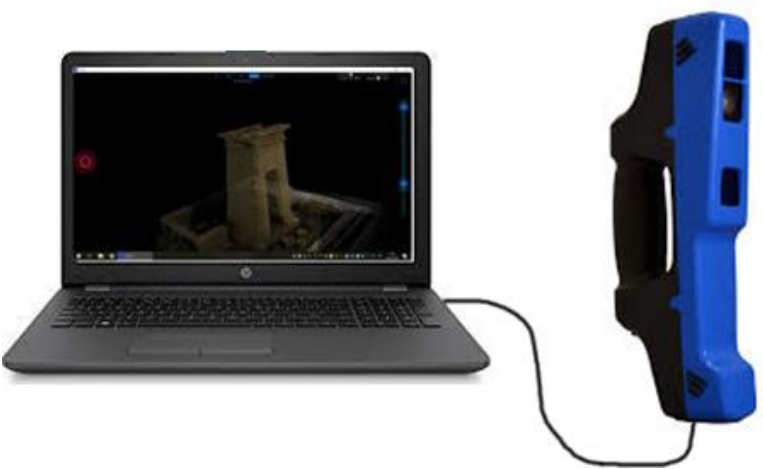

Figure 4. The F6 SR by Stonex connected via cable to a PC

The F6 SR is able to operate at a declared minimum distance of $250 \mathrm{~mm}$ with a certified accuracy of $90 \mu \mathrm{m}$ acquiring 640,000 points per second. Moreover, this device is equipped with a RGB camera and it is able to generate a photographic texture. The cable connection to a device (PC or tablet) enable to manage all the acquisition process in the free Mantis Vision Echo software (build 1.3.0), from the customization of some parameters (like camera and IR settings, range distance) to the visualization of the ongoing point cloud acquisition (different modes are available such as infrared, video, 3D). The F6 SR works with an emitter projecting a near infra-red (NIR) light through Mantis Vision's proprietary pattern onto the scene while a receiver calculates the distance of each mapped point through triangulation algorithm and of the 3D scene by stereoscopic parallax. The NIR solution enables to operate in any lighting conditions without the employment of markers.

\begin{tabular}{|l|l|}
\hline Model & F6 SR \\
\hline Speed & $640,000[\mathrm{pts} / \mathrm{s}]$ \\
\hline Range distance & $250-500[\mathrm{~mm}]$ \\
\hline Accuracy & $90 \mu \mathrm{m} \mathrm{@} \mathrm{250} \mathrm{mm}$ \\
\hline Resolution & $0.4 \mathrm{~mm} \mathrm{@} 250 \mathrm{~mm}$ \\
\hline RGB camera resolution & $1.3[\mathrm{MPx}]$ \\
\hline Video frame rate & $8[$ frame/s] \\
\hline
\end{tabular}

Table 2. F6 SR main specifications

Each NIR frame is strictly related to a video frame, in fact the software registers at the same time the colour with the RGB camera and the depth with the NIR receiver and associates to each point the colour extracted from the video. So, the "online registration" algorithm associates colour and geometry converting these two data into one coloured "point cloud frame" and registering frame by frame the 3D point cloud online and in real time. The result is a coloured point cloud composed by one or more groups containing one or more segments composed by several point cloud frames that are portions of the global point cloud. During the acquisition the software generates separated groups and segments if the algorithm loss the connection between one frame and the subsequent. The software operates a raw alignment of the point cloud frames through the online registration in real time and does not stop the scan if the registration between frames fails; the correct alignment of all frames can be completed in the post-processing phase. The operator moves the handheld device with free and slow motion around the object. It is possible to acquire a single scan or, if necessary, more than one and then co-register them in the post-processing phase. The right portion of the Temple of El-Hilla has been acquired with a single scan in about 10 minutes (about 5 minutes spending for the effective acquisition and 5 minutes for the instrument preparation). Thanks to the operator expertise, the acquired point cloud was composed by a single group containing one segment with 2214 frames. Nonetheless some misalignment between frames are stressed (Figure 5) but the point cloud can be re-register and edited in the processing phase.

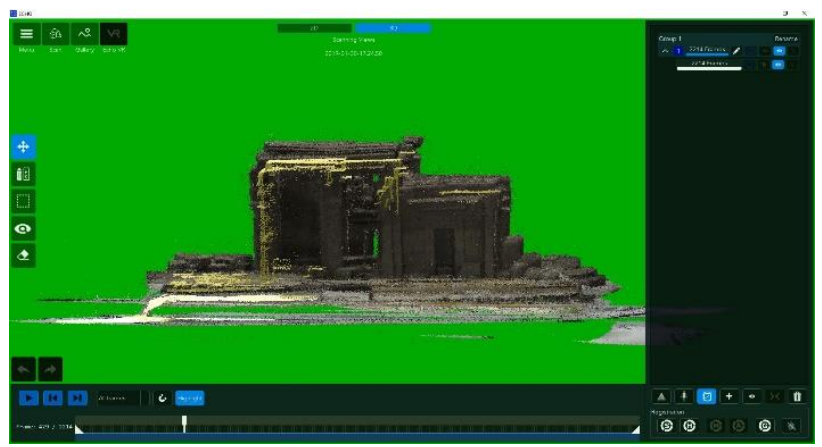

Figure 5. Misalignments between point cloud frames are visible after the acquisition, in yellow a single point cloud frame is highlighted

\section{DATA PROCESSING}

The acquired datasets have been processed according to standard workflows but customized to the needs as follows.

\subsection{Close-range photogrammetry}

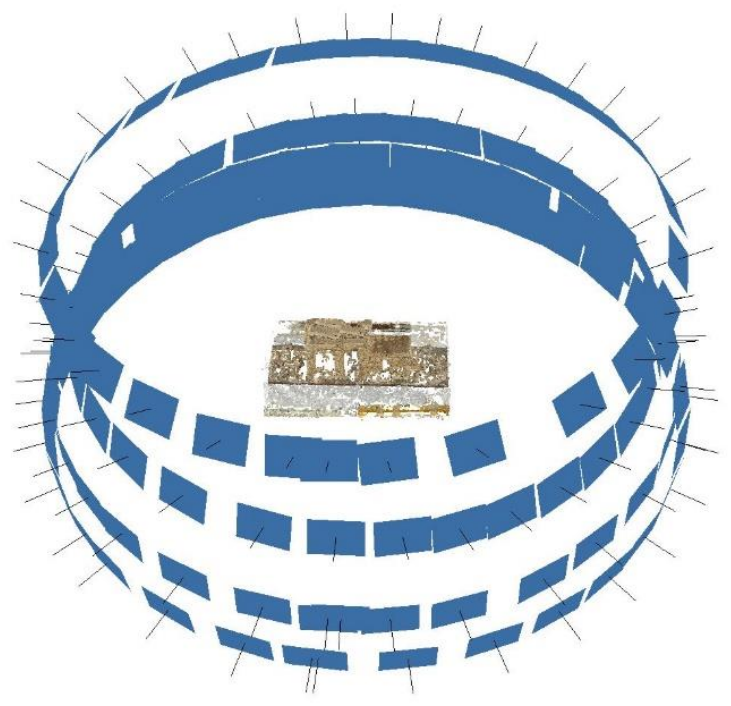

Figure 6. Image alignment and sparse cloud of tie points

The acquired digital images have been processed with the SfM-based software Agisoft Photoscan (currently known as Agisoft Metashape from the new build). Before proceeding with the image alignment and sparse cloud generation (Figure 6 ), a mask has been applied on each image with manual and semi-automatic procedures, in order to exclude the background. In fact, the context is stationary respect to the maquette due to the movement of the platform and if not masked it causes wrong photos alignment. With this strategy, the tie points extraction has been performed considering only the areas of each image with the maquette and the scale bars (Figure 7). 

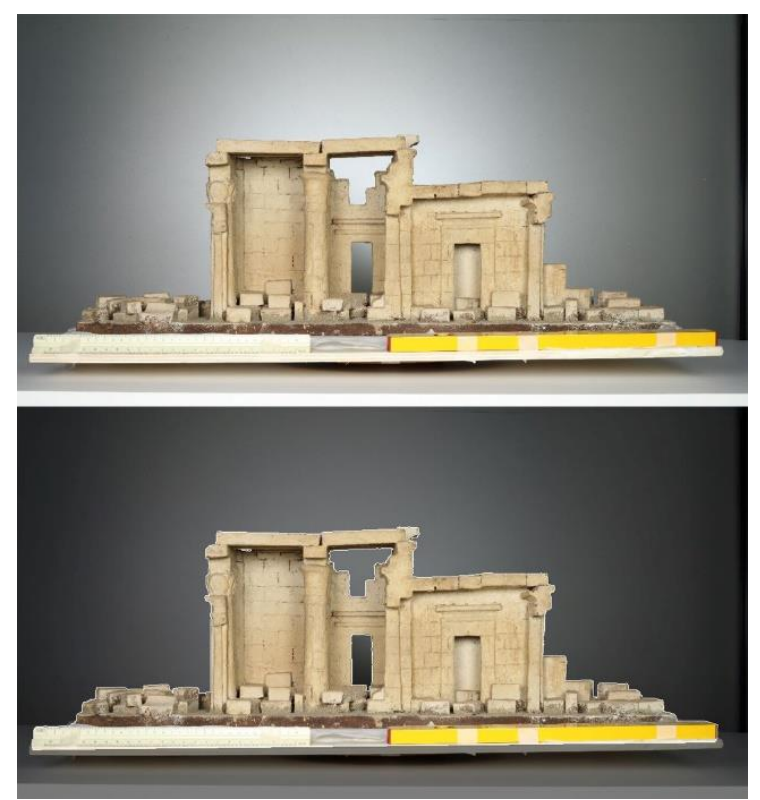

Figure 7. Image of the model of the Temple of El-Hilla (top) and the same image after the masking process (bottom)

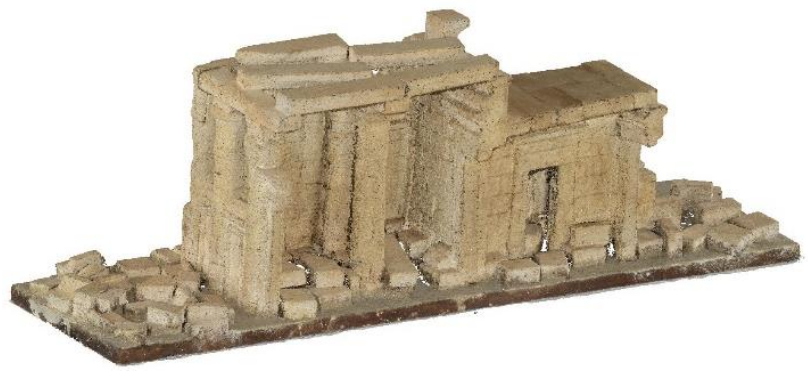

Figure 8. Photogrammetric dense point cloud

\begin{tabular}{|l|l|}
\hline $\mathrm{N}^{\circ}$ of processed images & 126 \\
\hline Tie points & 255,250 \\
\hline Dense cloud & $16,802,029$ \\
\hline Estimated GSD & $0.12[\mathrm{~mm} / \mathrm{px}]$ \\
\hline Reprojection error & $0.477[\mathrm{px}]$ \\
\hline Triangles of TIN & $\approx 1,000,000$ \\
\hline Wire dimension of TIN & $1-2.5[\mathrm{~mm}]$ \\
\hline
\end{tabular}

Table 3. Main details of the photogrammetric process

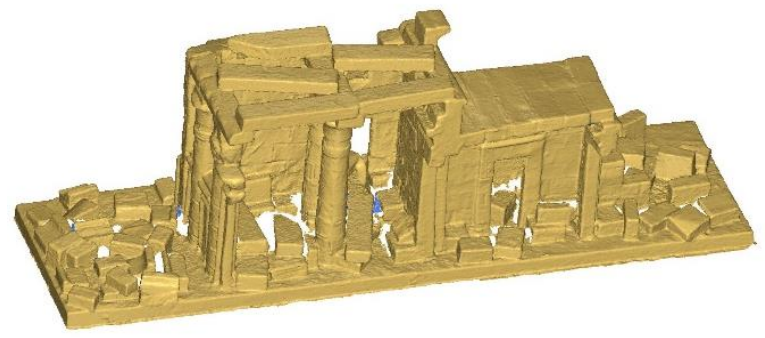

Figure 9. Photogrammetric TIN

Subsequently, high dense coloured point cloud of the maquette has been generated (Figure 8), composed by more than 16 million of points with an approximate volume of $0.11 \mathrm{~m}^{3}$ (Table 3). The three scale bars, previously positioned on the acquisition stage, have been used in order to scale the final dense point cloud. Finally, the Triangulated Irregular Network (TIN) has been created (Figure 9) with about 1 million triangles and wire dimension with a range of 1-2.5 mm.

\subsection{Stonex F6 SR: Echo Mantis}

For the F6 SR scans processing, the present research follows the workflow proposed by the manual: check of the acquired data, "High Quality (HQ) registration", editing, global registration, denoise, TIN generation.

In the acquisition phase Echo saves a project within the software containing a .mvx file where all the information about the point cloud and the video are stored. Only after the processing phase the point cloud and the TIN can be exported and edited in another software. After the acquisition, the point cloud obtained with the online registration requires the check of the operator consisting in the removal of groups and segments with only few frames. If a single scan contains more than one group and more than one segment for each group, it is necessary to re-run the registration algorithm to obtain a single group, that means that all the frames are co-register and aligned. Echo offers different algorithms (sequential, HQ, manual, automatic, global) to register frames, segments, groups and different scans also. After that, manual editing is suggested to remove unwanted portion of the point cloud before the global registration. This algorithm converts all the frames of one or more segments in a point cloud with a unique coordinates system, it exploits both geometric and radiometric data to rectify not visible misalignments and cumulative registration errors but requiring more calculating time. Then, two algorithms for denoise process are available, the Statistical Outlier Removal (SOR) to clean the point cloud and Moving Least Squares (MLS) to smooth it. Finally, it is possible to generate a TIN choosing between four different resolution (low, medium, high, custom) and additional settings about colour.

In the Temple of El-Hilla scan, the existence of only one group with one segment has allowed to skip the check step necessary to select groups and segments and has reduced the processing time. In fact, the global registration can be performed only on a single group. If the raw scan contains a lot of groups and a lot of frames, the processing time and the operator contribution increase to achieve a single group. In this case, the HQ registration has been directly employed to correct the misalignment visible in the raw data. This algorithm differs from the online registration employed during the acquisition because consists in a more time spending comprehensive process, this means that it considers all frames, segments and groups together, while the online registration processes data frame by frame (Figure 10).

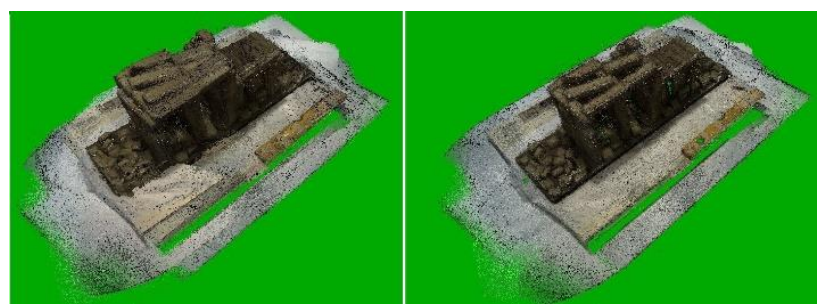

Figure 10. Point cloud after processing of the algorithm: online registration (left), HQ registration (right)

After that, the global registration algorithm converted the point cloud frames in a single point cloud with unique coordinates system. After a manual editing to remove the context of the acquired scene, the SOR algorithm reduced the noise of the point cloud (Figure 11). 

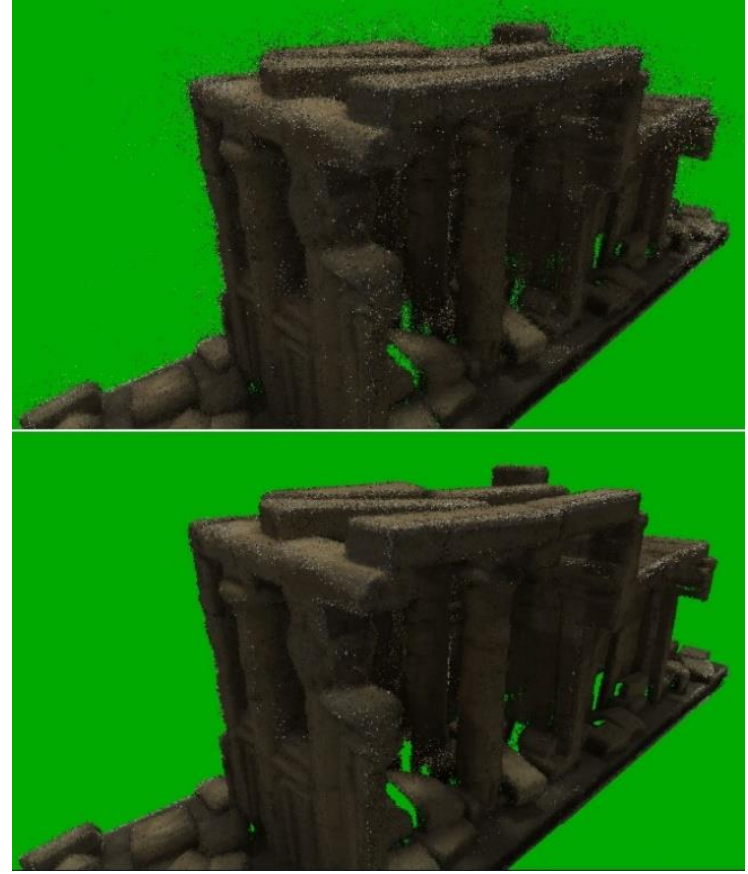

Figure 11. Point cloud before (top) and after (bottom) the SOR algorithm
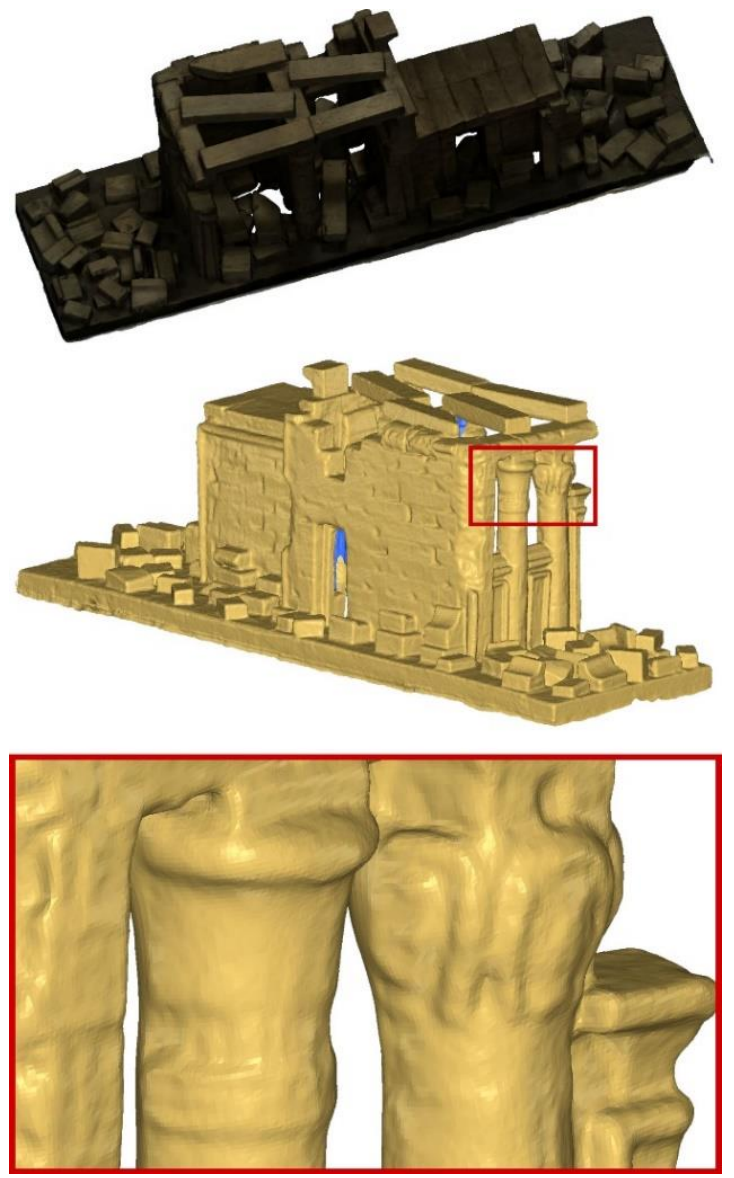

Figure 12. TIN of the Temple of El-Hilla obtained with F6 SR: with texture (top), without (centre) and zoom on the capitals (bottom)

Finally, the TIN has been created with high quality obtaining a 3D model ( Figure 12) with 2687064 triangles textured exploiting the video frames. In Table 4 the main characteristic of the F6 point cloud and TIN are reported: a big reduction of the number of points in the point cloud is due to the manual editing, but also to the SOR denoising; the TIN is composed by triangles with a wire of millimetre size.

\begin{tabular}{|l|l|l|}
\hline \multirow{2}{*}{$\begin{array}{l}\text { Point cloud } \\
\text { [pts] }\end{array}$} & Raw & $79,151,358$ \\
\cline { 2 - 3 } & After manual editing & $47,232,989$ \\
\cline { 2 - 3 } & After SOR algorithm & $44,730,529$ \\
\hline \multirow{2}{*}{ TIN } & Triangles & $2,687,064$ \\
\cline { 2 - 3 } & Wire dimension [mm] & $\approx 1$ \\
\hline
\end{tabular}

Table 4. Main characteristics of the F6 SR results

\section{COMPARISON BETWEEN DATASETS}

As regards the point cloud obtained from photogrammetric dataset, a number of neighbours density analysis has been performed with the open-source software Cloud Compare and it is possible to observe in Figure 13 a homogeneous density, this means that points are distributed uniformly on the surface of the maquette. The estimated mean value is ca. 73 points inside a sphere of radius $1 \mathrm{~mm}$ with a relatively low standard deviation (Figure 14). The areas with lower density are located in proximity of lacks of data due to various occlusions (e.g. the blocks).

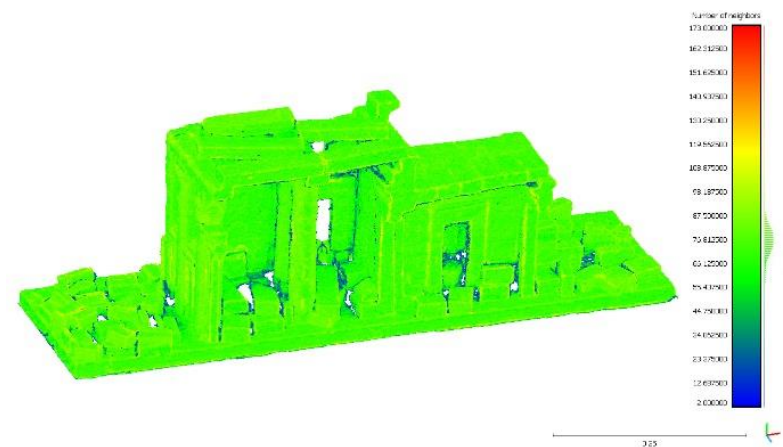

Figure 13. Density analysis of the photogrammetric point cloud

Gauss: mean $=73.259254 /$ std.dev. $=12.529427$ [4118 classes]

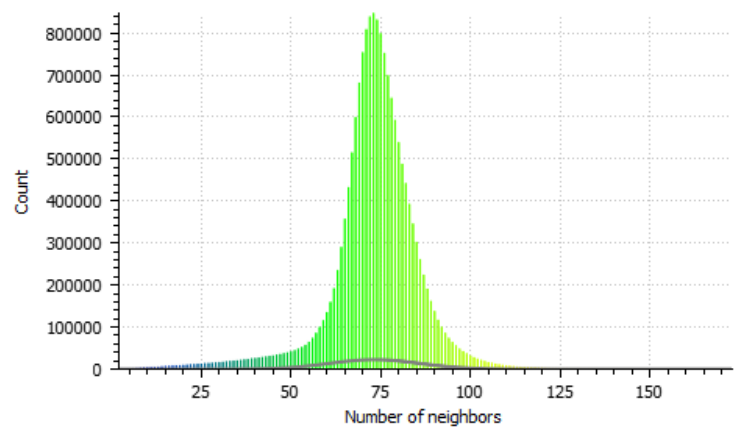

Figure 14. Density analysis histogram of the photogrammetric point cloud

As regards the point cloud acquired with Stonex F6 SR, a higher density is observed (Figure 15), ca. 291 points in a sphere of $1 \mathrm{~mm}$ (Figure 16) but, on the contrary of the photogrammetric dense cloud, the density is very not homogeneous. The upper parts of the model are those that present higher value (with peak of more than 700 points/V. sphere $r=1 \mathrm{~mm}$ ) due to the distance of acquisition and to the time spent by the operator on that portion. 
Thanks to free motion allowed by the handheld solution, the F6 SR point cloud shows reconstructed geometry more completed than the photogrammetric point cloud, also due to the restricting use of four fixed position on a tripod. The presence of projections and recesses generates occlusions, and consequently holes, especially on pavement and ceiling, uneasy to survey for the photogrammetric approach. This problem can be solved increasing the number of photos at the expense of acquisition and processing times. Nonetheless, some holes are present also in the F6 SR data, especially as regards inner chambers with small openings. The radiometric quality of the texture is visually more appreciable in the TIN from photogrammetric dataset, firstly because the full frame camera offers a 50.3 Megapixels sensor that is about fifty times bigger than the F6 SR one and secondly due to the improvement of natural lighting condition with LED panels during the photogrammetric acquisition. During the F6 SR recording is difficult to preserve an equal intensity on the model of light moving around the laser, also because of the presence of occlusions generating shadows.

In case of museum collection, as hereby presented, further observations are essential to for the purposes of standardization process. Even if called movable heritage, only few pieces are easy movable because of their fragility and inestimable values. The photogrammetric approach exploiting a rotating platform can drastically reduce the time of acquisition if the element can be moved, otherwise the camera with tripod needs to be repositioned for each photo with the lighting panels and the focus adjusted as also stated in Guidi et al., 2017. The rotating platform with fixed set solution cannot be replicated in every case and condition, also because the set requires an adequate space all around the masterpiece. Thus, the F6 SR constitutes a more suitable solution, limited only by its dimensions $(32 \times 12 \times 4.5 \mathrm{~cm} 3)$ and operating range $(25-50$ $\mathrm{cm})$.
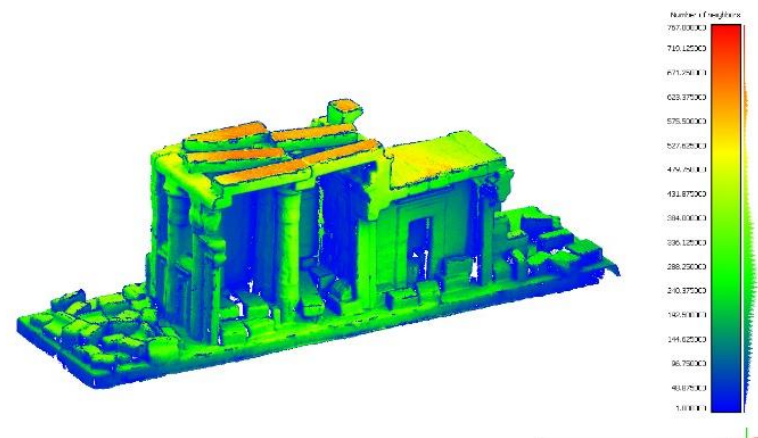

Figure 15. Density analysis of the F6 SR point cloud

\section{CONCLUSIONS AND FUTURE PROSPECTIVES}

The main aim of this experience tries to evaluate the suitability of a full frame camera and a new structured light laser in the framework of movable heritage documentation and 3D modelling. From the analysis of the two different workflows, considering an architectural maquette belonging to "Museo Egizio" of Torino as case study, a comparison between the two obtained dense point clouds and the texturized TIN has been considered evaluating different characteristic in terms of completeness and resolution of the two datasets. The Table 5 summarises the competitiveness of each approach in terms of time spending, suitability and costs as regard the specific case study.

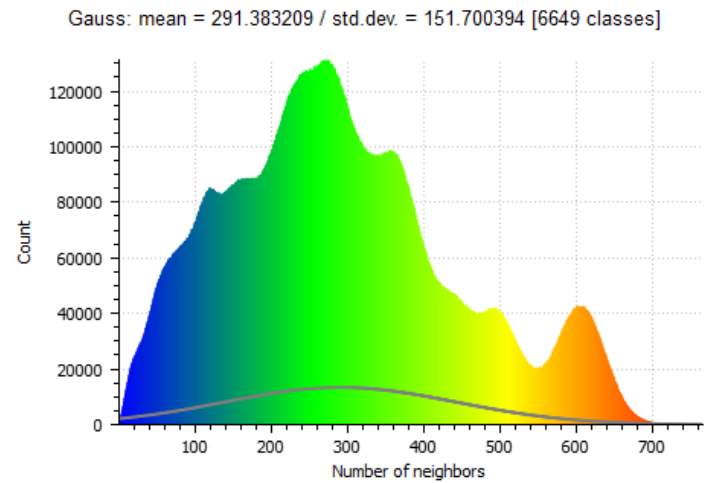

Figure 16. Density analysis histogram of the F6 SR point cloud

\begin{tabular}{|c|c|c|}
\hline Sensor & CANON & F6 SR \\
\hline Data acquired [GB] & $\begin{array}{c}7 \\
(. c r 2)\end{array}$ & $\begin{array}{c}10 \\
(. \mathrm{mvx})\end{array}$ \\
\hline $\begin{array}{l}\text { Time of acquisition } \\
\text { [min] }\end{array}$ & 35 & 10 \\
\hline $\begin{array}{l}\text { Dense point cloud }\left[\mathrm{N}^{\circ} \text { of }\right. \\
\text { points] }\end{array}$ & $16,802,029$ & $44,730,529$ \\
\hline Time of processing & & \\
\hline Completeness & & \\
\hline Resolution & & \\
\hline Texture quality & & \\
\hline Sensor cost & & \\
\hline
\end{tabular}

Table 5. Comparison between model obtained by Canon and by F6 SR

In this test field the F6 SR has demonstrated to be competitive for time of acquisition and processing, but also completeness and resolution, while the texture requires some improvements. As future perspectives, a deeper analysis is required to evaluate the accuracy of the F6 SR data comparing it with measured control and check points. Further case studies are necessary to test the F6 SR capabilities in case of movable heritage with different texture, materials, shape and dimensions. Objects smaller than the Temple of El-Hilla with dark texture represent challenge test fields for the F6 SR and requires more expertise and work, especially for data processing. It is suggested to test the potentiality of the different registration algorithms to fix scans with more than one group and to co-register different scans. Furthermore, solving the changes in lightning condition during acquisition phase can improve overall quality of final generated texture. The possibilities of the obtained results are not confined only to $3 \mathrm{D}$ visualisation, in fact they represent a valuable tool in terms of scientific analysis, museum management, cultural dissemination, online sharing and digital interrogation, also considering that museum collections are sometimes stored in depots and not visible, as the maquettes of the Nubian temples. In this framework the F6 SR potentiality could be very interesting for the rapid digitization of movable objects.

\section{ACKNOWLEDGEMENTS}

The authors would like to thank Alessia Fassone, the collection manager team of Museo Egizio di Torino for their availability and courtesy and the colleagues of Back to the Future project. They also declared the GAMHER research project (financed by PRIN 2015) as supporter. 


\section{REFERENCES}

Adami, A., Balletti, C., Fassi, F., Fregonese, L., Guerra, F., Taffurelli, L., and Vernier, P., 2015. The bust of Francesco II Gonzaga: from digital documentation to 3D printing. In: ISPRS Annals of Photogrammetry, Remote Sensing and Spatial Information Sciences, Vol. II-5/W3, pp. 9-15. https://doi.org/10.5194/isprsannals-II-5-W3-9-2015

Allegra, D., Gallo, G., Inzerillo, L., Lombardo, M., Milotta, F. L. M., Santagati, C., and Stanco, F., 2017. Hand Held 3D Scanning for Cultural Heritage: Experimenting Low Cost Structure Sensor Scan. In: Ippolito, A., (ed.), Handbook of Research on Emerging Technologies for Architectural and Archaeological Heritage, Chapter 16, IGI Global, Hershey, Pennsylvania, USA, pp. 475-499. https://doi.org/10.4018/978-1-5225-0675-1.ch016

Balletti, C., Costa, M., Guerra, F., Martinello, F., and Vernier, P., 2018. Modern and contemporary cultural heritage documentation and knowledge by surveying and its representation. In: International Archives of the Photogrammetry, Remote Sensing \& Spatial Information Sciences, Vol. XLII-2, pp. 63-67. https://doi.org/10.5194/isprs-archives-XLII-2-63-2018

Donadio, E., 2018. 3D photogrammetric data modeling and optimization for multipurpose analysis and representation of Cultural Heritage assets. Ph.D. Thesis, Politecnico di Torino, Torino, Italy, 2018.

Donadio, E., Sambuelli, L., and Spanò, A., 2018. ThreeDimensional (3D) Modelling and Optimization for Multipurpose Analysis and Representation of Ancient Statues. In: Gonzalez-Aguilera, D., (ed.), Latest Developments in Reality-Based 3D Surveying and Modelling, MDPI Books, Basel, Switzerland, pp. 95-118. https://doi.org/10.3390/books978-3-03842-685-1-5

Dondi, P., Lombardi, L., Malagodi, M., and Licchelli, M, 2017. 3D modelling and measurements of historical violins. In: Acta IMEKO, Vol. 6, $\mathrm{N}^{\circ}$ 3, Article 5, pp. 29-34. https://doi.org/10.21014/acta_imeko.v6i3.455
Guidi, G., Malik, U. S., Frischer, B., Barandoni, C., and Paolucci, F, 2017. The Indiana University-Uffizi project Metrological challenges and workflow for massive 3D digitization of sculptures. In: Proceedings of the $201723^{\text {rd }}$ International Conference on Virtual System \& Multimedia (VSMM), pp. 1-8. https://doi.org/10.1109/VSMM.2017.8346268

Kersten, T. P., Lindstaedt, M., and Starosta, D., 2018. Comparative Geometrical Accuracy Investigations of Handheld 3D Scanning System - an Update. In: International Archives of the Photogrammetry, Remote Sensing and Spatial Information Sciences, Vol. XLII-2, pp. 487-494. https://doi.org/10.5194/isprs-archives-XLII-2-487-2018

Lo Turco, M., Piumatti, P., Rinaudo, F., Tamborrino, R., and González-Aguilera, D., 2018. B.A.C.K. TO T.H.E. F.U.T.U.RE. - Bim Acquisition as Cultural Key TO Transfer Heritage of ancient Egypt For many Uses To many Users Replayed. In: Bertocci, S., (ed.), Programmi multidisciplinari per l'internazionalizzazione della ricerca. Patrimonio culturale, Architettura e Paesaggio. DIDA Press, Firenze, Italy, pp. 107-109.

Marziali, S., and Dionisio, G., 2017. Photogrammetry and macro photography. The experience of the MUSINT II Project in the 3D digitizing process of small size archaeological artifacts. In: Studies in Digital Heritage, Vol. 1, N 2, pp. 298 309. https://doi.org/10.14434/sdh.v1i2.23250

Patrucco, G., Chiabrando, F., Dondi, P., and Malagodi, M, 2018. Image and Range-Based 3D Acquisition and Modeling of Popular Musical Instruments. In: Proceedings from the Document Academy, Vol. 5, Iss. 2, Article 9. https://doi.org/10.35492/docam/5/2/9

Povroznik, N., 2018. 3D Models of Ancient Greek Collection of the Perm University History Museum Creation and Use. In: Ioannides, M., (ed.), Digital Cultural Heritage, Springer International Publishing, Cham, Switzerland, pp. 144-154. https://doi.org/10.1007/978-3-319-75826-8 